\title{
TITLE:
}

\section{Tricriticality in thermoreversible gels}

$\operatorname{AUTHOR}(\mathrm{S})$ :

Tanaka, Fumihiko; Matsuyama, Akihiko

\section{CITATION:}

Tanaka, Fumihiko ... [et al]. Tricriticality in thermoreversible gels.

Physical Review Letters 1989, 62(23): 2759-2762

ISSUE DATE:

1989-06-05

URL:

http://hdl.handle.net/2433/49179

RIGHT:

Copyright 1989 American Physical Society. 


\title{
Tricriticality in Thermoreversible Gels
}

\author{
Fumihiko Tanaka and Akihiko Matsuyama ${ }^{(a)}$ \\ Department of Physics, Faculty of General Education, Tokyo University of Agriculture and Technology, \\ Fuchu, Tokyo 183, Japan \\ (Received 30 January 1989)
}

\begin{abstract}
A simple model is introduced to describe the observed coexistence of gelation and phase demixing in atactic polystyrene solutions. It is shown that the multiple equilibria conditions for molecular clustering caused by physical cross linking can lead to a characteristic feature of the phase diagram. The condition is examined for the appearance of new criticai phenomena, such as a tricritical point or a triple point (or a three-phase equilibrium), as a result of interference between gelation and phase separation.
\end{abstract}

PACS numbers: $82.70 . \mathrm{Gg}, 64.60 . \mathrm{Kw}, 64.75 . \mathrm{tg}$

Recent experimental studies ${ }^{1,2}$ have furnished strong evidence that the gelation of atactic polystyrene (at-PS) in the solvent of carbon disulfide is thermally reversible, and also that the gel melting temperature agrees with the gel freezing temperature. It was also observed that apparently inactive at-PS can gel in a series of solvents including toluene and tetrahydrofuran. The physical gelation of at-PS can therefore be universal. The current understanding of the experimental observations is based on the co-occurrence ${ }^{3}$ of sol-gel transition and two-phase separation on the temperature-concentration plane. The purpose of the present paper is to derive the observed phase diagram from a molecular point of view, and to predict new phenomena caused by the interference between the two intrinsically different phase transitions: gelation and phase separation.

Consider a system of identical molecules dissolved in a solvent, each carrying functional groups. The functional groups on a molecule are assumed to be identical for simplicity and capable of forming physical bonds by pairwise association. The potential barrier of the cross linking considered here is of the order of thermal energy, so that bonding-unbonding equilibrium is easily established by thermal activation.

In thermal equilibrium, an intermolecular reaction yields polydisperse molecular aggregates, which we call "cluster" in the following. The cluster distribution is thermally controlled and strongly dependent on the total solute concentration. To derive the size distribution of such clusters, we consider the thermodynamics of the system.

The free energy of our system can be constructed by the sum of the two terms, $F=F_{\text {rea }}+\Delta F_{\text {mix }}$, each required in two different steps starting from the reference state where pure solvent and unreacted molecules are prepared separately. Here $F_{\text {rea }}$ is the free-energy change required to form the clusters from the reference state by pairwise connection of the functional groups. It is written as $F=\Sigma_{m} N_{m} \mu_{m}^{0}$ in terms of the chemical potential $\mu_{m}^{0}$ (or equivalently the internal free energy) of a single isolated $m$ cluster. The second term $\Delta F_{\text {mix }}$ describes the free- energy change required in the process of mixing thus constructed clusters with the solvent. According to the lattice theory developed by Flory and Huggins, ${ }^{4}$ it is given by

$$
\beta \Delta F_{\text {mix }}=N_{0} \ln \phi_{0}+\sum_{m=1}^{\infty} N_{m} \ln \phi_{m}+\Omega \chi \phi(1-\phi),
$$

where $\phi_{0}$ is the volume fraction of the solvent, $\phi_{m}$ is the volume fraction of the $m$ clusters, $\beta \equiv 1 / k_{B} T$ is the inverse temperature, $\Omega$ is the total number of lattice cells in the system, and $\chi$ is the solvent-solute interaction parameter. The total solute concentration is given by $\phi$ $=\Sigma \phi_{m}$.

In thermal equilibrium, each molecule is in chemical equilibrium through bonding and unbonding processes. Therefore, since $\mu_{m} / m$ is independent of $m$, this imposes the following multiple-equilibria condition: ${ }^{5}$

$$
\mu_{m} / m=\mu_{1},
$$

where $\mu_{m} \equiv\left(\partial F / \partial N_{m}\right)_{T, N_{m}}$ is the chemical potential of an $m$ cluster. Substitution of the specific form for $\mu_{m}$ derived from $F$ yields $\phi_{m}=K_{m} \phi_{1}^{m}$ for the volume fraction of $m$ clusters, where the association constant $K_{m}$ is expressed as $K_{m}=\exp \left(m-1-\Delta_{m}\right)$ in terms of the reduced free-energy difference $\Delta_{m}$ defined by $\Delta_{m} \equiv \beta\left(\mu_{m}^{0}-m \mu_{1}^{0}\right)$. The free-energy gain per single molecule by participating in an $m$ cluster from isolation is given by $\delta_{m}=\Delta_{m} / m$. Thus the free energies $\Delta_{m}$ of cluster formation determine the entire distribution of aggregates. The total concentration is now given by a power series of $\phi_{1}$, the unimer concentration, the $m$ th power of which is accompanied by the association constant $K_{m}$. Application of the Cauchy-Hadamard theorem gives the convergence radius $\Phi$ of the power series in the form $1 / \Phi=\overline{\lim }\left(K_{m}\right)^{1 / m}$ $=\exp \left(1-\delta_{\infty}\right)$, where the least upper bound of the limit has been indicated by a bar. The quantity $\delta_{\infty}$ is the limiting value of $\delta_{m}$ as $m \rightarrow \infty$, i.e., the free-energy gain per molecule for the formation of an infinite (macroscopic) cluster. Within the radius of convergence, the equation $\phi=\sum \phi_{m}$ gives a one-to-one relationship between $\phi$ and $\phi_{1}$. By inverting this relation, let us express the unimer 
concentration in terms of the total concentration: $\phi_{1}$ $=\psi(\phi)$, for $0 \leq \phi_{1}<\Phi$. The function $\psi(\phi)$ is a monotonically increasing function of $\phi$ since all the coefficients $K_{m}$ are positive definite.

In the case where $\delta_{m}$ is a monotonically decreasing function, the cluster size $m^{*}$ at which fraction $\phi_{m}$ takes maximum for a given $\phi_{1}$ is infinite and a macroscopic cluster appears as soon as $\phi_{1}$ exceeds the critical value $\phi_{1}^{*} \equiv \exp \left(\delta_{\infty}-1\right)$. Hence we have gelation. The total concentration $\phi^{*}$ obtained from $\phi_{1}^{*}$ gives the concentration at which sol-to-gel transition occurs. It depends on the temperature through $\delta_{\infty}$. For $\phi_{1}$ above $\phi_{1}^{*}$, the summation of the power series does not reach $\phi$, since it cannot accommodate the contribution from the infinite network. The excess amount $\phi-\Sigma \phi_{m}$ is consumed to form a macronetwork. We thus have a decomposition of the total concentration into $\phi_{S}=\Sigma \phi_{m}$ for the sol component and $\phi_{G}=\phi-\phi_{S}$ for the gel component. The chemical potential $\mu_{G}$ for a single molecule participating in the gel network can be found by taking the $\lim \left(\mu_{m} / m\right)$. The global uniformity of the molecular chemical potential imposes an additional condition $\mu_{G}=\mu_{1}$. By using the specific form for $\mu_{m}$ derived from the free energy $F$, we find that the unimer concentration remains fixed at $\phi_{1}^{*}$ above the gelation threshold, while the total concentration increases. This is a simple picture of gelation ${ }^{6}$ based on the molecular-field approximation.

To proceed a step further and obtain specific results, we must introduce a model for the internal structure of a cluster. Here, we consider the simplest model in which a cluster is constructed in the form of Kayley (acyclic) tree of $f$-functional molecules. Intracluster loop formation is neglected. This is a crude approximation based on the classical molecular-field picture of gelation. ${ }^{6,7}$

The internal partition function $Z_{m}$ of a single $m$ cluster can be obtained by combinatorial counting as

$$
Z_{m}=\left[W_{m}(f) / m !\right] p^{m-1}(1-p)^{f m-2 m+2},
$$

where $p$ is the probability of bond formation for a pair of active groups and $W_{m}(f)$ is the number of combinations in which $m$ molecules form a tree. This combinatorial factor depends on the structure of a molecule and is given by ${ }^{6} W_{m}(f)=(f m-m) ! f^{m} /(f m-2 m+2)$ !, provided that the $f$-functional groups on a molecule are indistinguishable. For a long flexible chain carrying a large number of active side groups, the functionality $f$ is of the order of $n$, the degree of polymerization, and the factor $f^{m}$ in $W_{m}$ must be omitted since the side groups can be sequentially numbered.

The partition function $Z_{m}$ gives the free-energy change $\delta_{m}$ of our concern through the relation $\exp (-m$ $\left.\times \delta_{m}\right)=Z_{m} / Z_{1}^{m}$ by definition. It is in fact a monotonically decreasing function of $m$ and approaches a limiting value

$\delta_{\infty}=1-(f-1) \ln (f-1)+(f-2) \ln (f-2)-\ln \lambda(T)$,

where the parameter $\lambda(T)$ is defined by $\lambda(T) \equiv e f p /$ $(1-p)^{2}$. The statistical weight of a bond formation relative to the weight of two unreacted functionalities is expressed as $p /(1-p)^{2}=\exp \left(-\beta \Delta f_{0}\right)$ in terms of the free-energy change $\Delta f_{0}$ of a single-bond formation by the law of mass action. Splitting the free energy into the entropy $\Delta s$ and energy $\Delta \epsilon$, we have $\lambda(T)=\lambda_{0} \exp (-\beta$ $\times \Delta \epsilon)$, where $\lambda_{0} \equiv e f \exp \left(\Delta s / k_{B}\right)$. Since all physical quantities depend on a combined variable $\lambda(T)_{\phi}$ instead of the concentration, we call $\lambda(T)$ the temperature shift factor.

According to the general prescription, ${ }^{6}$ the infinite series $\sum \phi_{m}$ can be explicitly summed up by introducing a parameter $\alpha$ defined by $\lambda \phi_{1} \equiv \alpha(1-\alpha)^{f-2}$. We find $\lambda \phi$ $=\alpha(1-f \alpha / 2) /(1-\alpha)^{2}$ within the radius of convergence. The limit of convergence is given by $\alpha_{c}=1 /(f-1)$, or equivalently

$$
\phi^{*}=1 / 2(f-2) \lambda(T) .
$$

This relation gives the sol-gel transition curve on the temperature-concentration plane.

In the following we present theoretical results on the phase behavior and the solution properties of the system. ${ }^{8}$ The following quantities are numerically calculated.

(1) Coexistence curves (binodals) of the phase equilibrium are derived by the coupled equations, $\mu_{0}\left(\phi^{\prime}\right)$ $=\mu_{0}\left(\phi^{\prime \prime}\right)$ and $\mu_{1}\left(\phi^{\prime}\right)=\mu_{1}\left(\phi^{\prime \prime}\right)$, where $\mu_{0}$ is the solvent chemical potential and $\mu_{1}$ is the chemical potential of an isolated molecule. Solute volume concentrations $\phi^{\prime}$ and $\phi "$ correspond to that of the lower and higher concentration phases, respectively. When these concentrations are higher than the threshold $\phi^{*}$, they are uniquely decomposed into the sol component and the gel component according to the scheme described above. The chemical potentials are then expressed as a function of the volume concentration of the two components.

(2) Spinodal lines are derived by the condition $\partial \Delta \mu_{0} /$ $\partial \phi=0$. This leads to

$$
\frac{1}{1-\phi}+\frac{\kappa(\phi)}{n \phi}-2 \chi=0 \text {, }
$$

where $\kappa(\phi)$ is defined by $\kappa(\phi) \equiv d \ln \psi(\phi) / d \phi$ when $\phi<\phi^{*}$ and $\kappa(\phi) \equiv 0$ when $\phi>\phi^{*}$. This $\kappa$ is a function of the combined variable $y \equiv \lambda \phi$ for our tree model and explicitly given by

$\kappa(y)=\left\{f\left(1+[1-2(f-2) y]^{1 / 2}\right)-2(f-2) y\right\} / 2(2 y+f)$.

(3) The osmotic pressure $\pi$ is proportional to the solvent chemical potential, $\pi \beta a^{2}=-\beta \Delta \mu_{0}$, and the number-average mean cluster size is given by $\langle m\rangle=\phi_{S}$ / $\int \kappa(\phi) d \phi$.

For the numerical calculation we take the temperature and the volume concentration as independent variables. The temperature is measured by a dimensionless deviation $\tau \equiv 1-\Theta / T$ from the unperturbed theta temperature $\Theta$ which satisfies an equation $\chi(\Theta)=\frac{1}{2}$. In terms of $\tau$, 

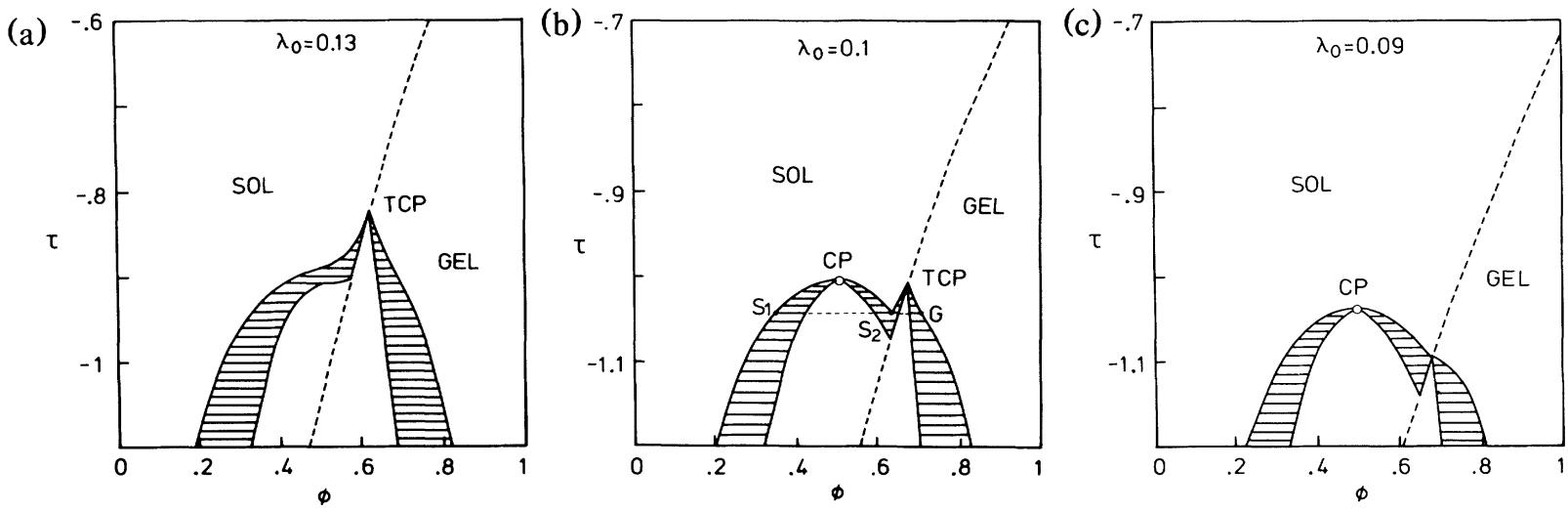

FIG. 1. Temperature-concentration phase diagram of low-molecular-weight thermoreversible gels.

the $\chi$ parameter and the shift factor can be expressed as $\chi=\frac{1}{2}-\psi_{1} \tau$ and $\lambda(T)=\lambda_{0} \exp [r(1-\tau)]$, where $r$ $\equiv-\Delta \epsilon / k_{B} \Theta$. We have three material parameters $\psi_{1}, \lambda_{0}$, and $r$. In addition to these three, we must specify the functionality $f$ and the number $n$ of the statistical units on a chain.

Figure 1 shows the calculated results for low-molecular-weight molecules. We have fixed $f=3, n=1$, and $r=1$ for a typical example. The entropy parameter $\lambda_{0}$ is changed from Fig. 1(a) to 1(c). Note that the scale of the temperature axis is magnified. We have one-phase sol, one-phase gel, and unstable region. The binodals and the spinodals are drawn. The shaded area between them is a metastable region. The point $\mathrm{CP}$ shows the critical solution point. As we show in Fig. 2, our molecular-field theory predicts that the reversible gelation is a second-order phase transition. Therefore the point where a gelation line meets a binodal at the top of the unstable region must be a tricritical point (TCP) [Figs. 1 (b) and 1(c)]. ${ }^{9,10}$ This new point appears as the result of the interference between the two intrinsically different phase transitions: gelation and phase separation.

As the entropy parameter $\lambda_{0}$ increases, we find in Fig. 1(b) another new phenomenon: a three-phase equilibri-

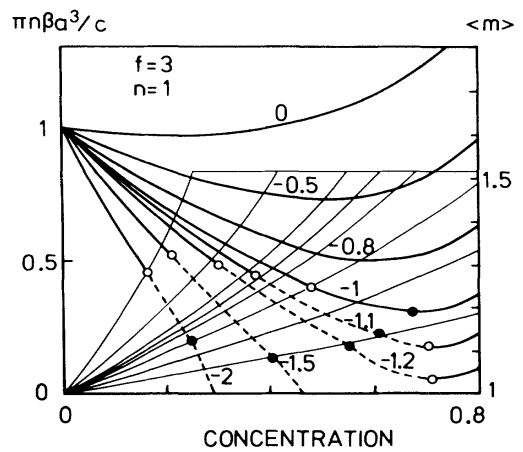

FIG. 2. Osmotic pressure and mean average cluster size are shown. Temperature is varied from curve to curve. um (or a triple point). Since the TCP moves upwards, two different sol phases $S_{1}$ and $S_{2}$ and a gel phase $G$ can simultaneously coexist. As $\lambda_{0}$ increases still further, the critical point disappears as shown in Fig. 1(c). We have a TCP only in the phase diagram. This phase diagram resembles that of the isotope mixture ${ }^{11}$ of liquid ${ }^{3} \mathrm{He}$ and liquid ${ }^{4} \mathrm{He}$.

Figure 2 shows the osmotic pressure and the numberaverage mean cluster size for $\lambda_{0}=0.1$. The temperature $\tau$ is varied from curve to curve. At the temperature where $\tau=0$, the second virial coefficient is actually negative as seen from the initial slope. The osmotic pressure has a kink at the gelation concentration shown by filled circles. The compressibility therefore jumps at the gelation point. Our approximation thus indicates that the gelation is a second-order phase transition. The open circles show the spinodal points. Between the two open circles on a line the system is unstable. The numberaverage cluster size $\langle m\rangle$ is a small number even at gelation.

Figure 3 shows the comparison of the theoretical calculation with the observed phase diagram ${ }^{2}$ for monodisperse at-PS in the solvent of $\mathrm{CS}_{2}$. The molecular weight of the at-PS is $3 \times 10^{4}$. Rheologically found gela-

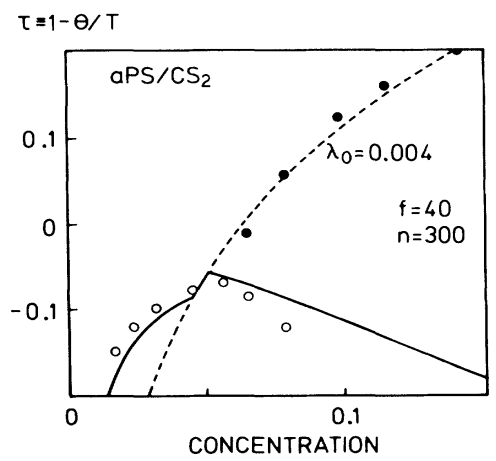

FIG. 3. Comparison of the calculated phase diagram with the experimental data for at-PS/CS 2 . 
tion points are indicated by filled circles. The thick line shows the calculated spinodal and the open circles show experimentally observed cloud points. The entropy parameter $\lambda_{0}$ is varied to fit the experimental data, while the energy parameter $r$ is fixed to be $r=4$ by using experimental values. ${ }^{2}$ The so-called "critical gel concentration $^{2}$ (CGC)," below which no gelation is observed, is not predicted in this theory. We cannot specify the functionality $f$ without ambiguity, since the molecular mechanism of the segment association has not been clarified. Here we present one speculation on the possible mechanism. In order for the polymer segment to be tightly bound, the large side groups must be on the same side of the backbone chain. Local isotacticity is required for this. Under such an assumption, the functionality $f$ must be proportional to the number of, for example, diad or triad on a chain. For a completely random chain, it is roughly given by the number which is 1 order of magnitude smaller than $n$.

In conclusion, we have shown that a simple molecular-field theory presented here can properly describe the global characteristics of the phase behavior of the thermoreversible gelation.

${ }^{(a)}$ Present address: Department of Physical Engineering, Faculty of Engineering, University of Tokyo, Tokyo 113,
Japan.

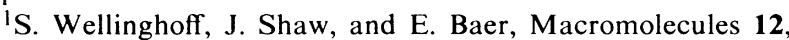
932 (1979).

${ }^{2}$ T. M. Tan, A. Moet, A. Hiltner, and E. Baer, Macromolecules 16, 28 (1983).

${ }^{3}$ P. G. de Gennes, Scaling Concepts in Polymer Physics (Cornell Univ. Press, Ithaca, 1979), Chap. 5; J.-F. Joanny, Polymer 21, 71 (1980); A. Coniglio, H. E. Stanley, and W. Klein, Phys. Rev. B 25, 6805 (1982); D. Stauffer, A. Coniglio, and M. Adam, Adv. Polymer Sci. 44, 103 (1982).

${ }^{4}$ P. J. Flory, Principles of Polymer Chemistry (Cornell Univ. Press, Ithaca, 1953), Chap. 12.

${ }^{5}$ For example, see J. N. Israelachvili, in Physics of Amphiphiles: Micelles, Vesicles and Microemulsions, edited by V. Degiorgio and M. Corti (North-Holland, Amsterdam, 1985), p. 24.

${ }^{6}$ W. H. Stockmayer, J. Chem. Phys. 11, 45 (1943); 12, 125 (1944).

${ }^{7}$ P. J. Flory, J. Am. Chem. Soc. 63, 3083 (1941); 63, 3091 (1941); 63, 3096 (1941).

${ }^{8}$ Calculation of the spinodals has been reported in F. Tana$\mathrm{ka}$, Macromolecules (to be published).

${ }^{9}$ C. M. Knobler and R. L. Scott, in Phase Transitions and Critical Phenomena, edited by C. Domb and J. L. Lebowitz (Academic, New York, 1984), Vol. 9.

${ }^{10}$ J. C. Wheeler and P. Pfeuty, J. Chem. Phys. 74, 6415 (1981); J. C. Wheeler, R. G. Petschek, and P. Pfeuty, Phys. Rev. Lett. 50, 1633 (1983).

${ }^{11}$ M. Blume, V. J. Emery, and R. B. Griffiths, Phys. Rev. A 4, 1071 (1971). 